außerordentlich schöne Photogramme. Bald darauf und unabhängig von Goldstein ist es in England von Curtis entdeckt und von Fowler ausigemessen: wordien. Dieses Heliumspektrum bildet ein lehrreiches Bindeglied zwischen dem Viellinienspektrum des Wasserstoffs und den typischen Bandenspektren. Es klärt dadurch indirekt das -Rätsel des Viellinienspektrums und ordnet dieses der Wasserstoffmolekel zu. Auch in quantitativer Hinsicht steht das Helium-Bandenspektrum in der Mitte zwischen idem Wasserstoff-Viellinienspektrum und z. B. 'den typischen Stickstoffbanden. Rechnet man nämlich mit Lenz nach der Theorie der quantenmäßigen Rotationen (Bjerrum, Schwarzschild, Heurlinger, Lenz) aus den Fowlerschen Messungen das Trägheitsmoment für den Träger dieses Spektrums aus, so findet man es etwa zehnmal so groß wie dasjenige der $\mathrm{H}_{2}$-Molekel im Vieliinienspektrum und etwa zehumal so hiein wie àsjenigo der $\mathrm{N}_{\text {y-Molekel }}$ in den sog. Cyanbanden.

Dem Chemiker gibt dieses He-Spektrum eive harte Nub zu knacken auf. Als Bandenspektrum gehört es zu einem molekularen Gebilde, einer $\mathrm{He}_{2}$-Molekel; ein He-Atom hat kein merkliches Trägheitsmoment. Wir sehen also hier zum ersten Male ein inertes Atom wenigstens vorübergehend eine Verbindung eingehen; das einatomige Helium verhält sich zeitweise zweiatomig. Daß auch die von Goldstein beschriebenen Anregungsbedingungen dieses He-Spektrums guit zu der Vorstellung einer instabilen oder metastabilen $\mathrm{He}_{2}$-Bindung passen, möge hier nur angedeutet werden. -

Goldstein hat seine wissenschaftliche Laufbahn in reinster Hingabe an das Phänomen, an die Licht- und Farbenerscheinung, durchlaufen. Kein wissenschaftlicher Ehrgeiz, kein Interesse am. Unterricht oder an dem der Erscheinung angegliederten farblosen Begriffssystem der Theorie hat ihn von seinem eigentlichen Pfade abgelenkt. Auch sein Interesse an der wissenschaftlichen Mitteilung in Wort und Schrift scheint weit zurückgetreten gegenüber seinem brennenden Interesse an der Mannigfaltigkeit der Erscheinungen. Ohne seiner Bescheidenheit zu nahe treten zu wollen, werden wir ein wenig an Goethes leidenschaftliche Hingabe an die visuelle Erscheinung und an seine Ablehnung der dahinter wirkendien kausalen Fäden erinnert. Und' so wollen wir unserem Jubilar, als Zusammenfassung seiner Arbeitsrichtung, die Worte von Lynkeus-Goethe in den Mund legen:

Ihr glücklichen Augen,

Was je ihr gesehn,

Es sei wie es wolle,

Es war doch so schön!

\title{
Die Untersuchungen Goldsteins über die Einwirkung der Kathodenstrahlen auf chemische Verbindungen.
}

\author{
Von W. Marckwald, Berlin.
}

Im Jahre 1894 machte Eugen Goldstein die überraschende Entdeckung, daß gewisse Alkalisalze, besonders die Halogenide, unter der Einwirkung von Kathodenstrablen charakteristische und intensive Färbungen annehmen. Chlornatrium färbt sich gelbbraun, Chilorkalium violett, Bromkalium blau. Diese ,Nachfarben" zeigen eine mehr oder minider große Beständigkeit, solange die gefärbten Salze vor Licht geschützt und bei gewöhnlicher Temperatur aufbewahrt werden. Erhitzen und Belichten bringt die Farbe schnell zum Verschwinden.

Anfangs schien die Eirscheinung auf eine kleine Zahl von Alkalisalzen beschränkt zu sein, bald aber konnte Goldstein zeigen, daß auch solche Salze, die zunächst keine Nachfarben im Kathodenlicht gegeben hatten, sich färbten, wenn sie vor der Bestrahlung geschmolzen oder doch hoch erhitzt worden waren. Nach dem Erkalten färbten sich unter der Einwirkung der Kathodenstrahlen zum Beispiel Kaliumsulfat grün, Natriumcarbonat rosa, Calciumchlorid lachsfarben, Baryumsulfat grünblau.

Eino zweite Klasse von Nachfarben zeigen die Salze, wenn sie während der Bestrahlung erhitzt werden. Sie nehmen dann nicht nur andere Farben an wie in der Kälte - so wird Natriumsulfat violett, Calciumchlorid graublau-, sondern die Nachfarbe ist dann auch viel beständiger sowohl gegen hohe Temperatur wie auch gegen Belichtung.

Diese zweite Klasse ron Nachfarben ist weniger studiert worden. Die erste Klasse aber hat das Interesse der Chemiker und Physiker in hohem $\mathrm{MaBe}$ in Anspruch genommen. Nach der Entdeckung des Radiums konnte Giesel zeigen, daß die von den radioaktiven Stoffen ausgehenden Strahlen die nämlichen Nachfarben an den Salzen hervorrufen wie die Kathodenstrahlen. Goldstein selbst fand ausgehend von der Hypothese, dab die Wirkung der Kathodenstrahlen wesentlich auf der Bildung uiltravioletten Lichtes bei ihrem Auftreffen auf Hindernisse beruht, daB ultraviolette Lichtstrahlen ebenfalls die Nachfarben der Salze hervorrufen.

Utber die Erklärung des Phänomens ist viel diskutiert worden. Der Umstand, daB Giesel ähnliche Färbungen von Alkalichloriden durch Einwirkung von Dämpfen der entsprechenden Metalle auf die Salze hervorrufen konnte, ließ vermuten, daß es sich um feste Lösungen der Metalle selbst oder von deren Subchloriden in den 
Chloriden handle. Diese Auffassung widerlegte Goldstein besonders dadurch, daB er. zeigte, da $B$ die vollkommen farblose Lösung seiner gefärbten Salze, die auch bei der Verdampfung weißes Salz hinterläßt, neutral reagiert, während die Lösung der Gieselschen Salze alkalische Reaktion zeigt. Ferner sind die Gieselschen Färbungen ungleich lichtbeständiger als die Nachfarben erster Klasse. Hingegen scheinen die Veränderungen, die die Salze bei der Einwirkung des Kathodenlichtes in der Hitze erleiden, und die durch die Bildung der Nachfarben zweiter Klasse in Erscheinung tritt, mit den Gieselschen Salzen übereinzustimmen. Denn jene Salze lösen sich in Wasser mit alkalischer Reaktion.

Eine weitere Auflkärung über das Phänomen brachte die Untersuchung gewisser Ammoniumsalze und organischer Halogenverbindungen. Wenn man mit flüssiger Luft abgekühltes Ammoniumchlorid der Einwirkung der Kathodenstrahlen aussetzt, so färbt es sich gelbbraun. Die Färbung ist höchst licht- und temperaturempfindlich. Ahnlich färbt sich Ammoniumjodid braun und Ammoniumfluorid tiefblau. Auch die durch organische Radikale substituierten Ammoniumsalze zeigen Nachfarben erster Klasse, so z. B Dimethylammoniumchlorid gelbgrün, Trimethylammoniumchlorid je nach der Temperatur gelblichrosa bis tiefgoldgelb, Tetramethylammoniumchlorid zitronengelb bis schwefelgelbgrün. Aber auch solche organischen Verbindungen, in denen das Halogen komplex gebunden ist, -wie Chlor- un'd Bromessigsäure, Bromoform, Chloral erleiden-bei der Temperatur der flüssigen Luft durch Kathodenstrahlen lichtempfindliche Färbungen, die hingegen bei den entsprechenden halogenfreien Verbindungen (Essigsäure, Aldehyd) ausbleiben. Könnte bei den Ammoniumsalzen allenfalls noch an die Bildung des Ammoniumradikals gedacht werden, so ist eine derartige Annahme bei den zuletzt genannten Verbindungen ausgeschlossen. Goldstein kommt daher zu dem Ergebnis, daß die Nachfarben erster K.lasse zeigenden Verbindungen, wenn die Färbungen überhaupt auf einer chemischen Veränderung und nicht nur auf einer Zustandsänderung der Stoffe beruhen, jedenfalls die Spaltungsstücke sebeneinander in fester Lösung enthalten. Dadurch würde sich die große Neigung zur Wiedervereinigung erklären.

Für die Auffassung, daß die gefärbten Salze allotrope Modifikationen der farblosen darstellen, spricht die Beobachtung Goldsteins, daß auch ein Element, der Schwefel, eine Nachfarbe erster Klasse zeigt, wenn er, in flüssiger Luft gekühlt, von Kathodenstrahlen getroffen wird. Er färbt sich chamois. Die Farbe verschwindet beim $\mathrm{Be}$ lichten selbst bei der Temperatur der flüssigen Luft in wenigen Minuten, bei Zimmertemperatur ist sie überhaupt nicht zu erhalten.

Nar eine kleine Zahl von Salzen, z. B. die Haloide der Alkalimetalle, gibt auch im Zustande höchster Reinheit die Nachfarben. Bei den meisten Salzen ist das Eintreten der Färbung an die Gegenwart spurenweiser Verunreinigungen geknüpft. Das konnte Goldstein zuerst am Kaliumsulfat nachweisen. Die Grünfärbung, die das käufliche Salz nach dem Schmelzen unter der Einwirkung der Kathodenstrahlen regelmäßig erzielt, bleibt aus, wenn das $\mathrm{Salz}$ mit größter Sorgfalt von Carbonat befreit wird. Ein kleiner Zusatz von Kaliumcarbonat zur Schmelze im Verhäitnis von $1: 10000$ genügt noch; um eine Färbung hervorzurufen. Es ist aber nötig, die Verunreinigung mit dem Salz zu verschmelzen. Ein bloßer Zusatz genügt nicht. Die Nachfarbe hängt von der Natur der Verunreinigung ab. So gibt Kaliumsulfat bei einem kleinen Zusatz von Kalliumchlorid und Natriumchlorid eine violette, von Lithiumchlorid eine blaugraue Nachfarbe. Die Nachfarben bieten also ein sehr empfindliches analytisches Hilfsmittel, um die Reinheit der Salze zu prüfen und eventuell die Art der Verunreinigung zu ermitteln.

Die Beeinflussung der Nachfarben durch spurenweise Verunreinigungen erinnert an die Wirkung, die ähnlich kleine und noch kleinere Verunreinigungen auf das Phosphoreszenzlicht gewisser Salze ausüben. Auch auf diesem Gebieto verdanken wir Goldstein interessante Beobachtungen. Das von dea Kathodenstrahlen hervorgerufene Phosphoreszenzlicht farbloser reiner Salze ist; wie er fand, in der Regel blau oder violett: Sehr geringe Beimengungen namentlich gefärbter Salze, z. B. solcher des Kupfers, Nickels, Kobalts, Mangans, aber rufen eine andersfarbige, kräftige. Lichtemission hervor, die häufig eine erhebliche Nachdauer hat. Hier genügen schon Beimischungen von weniger als $1: 10000000$ zur Erzielung einer sicher erkennbaren Wirkung.

In engster Beziehung zu den Nachfarben steht eine Erscheinung, die Goldstein bei der Untersuchung des Phosphoreszenzspektrums aromatischer Verbindungen. bei der Temperatur der flüssigen Luft im Kathodenlicht beobachtete. Die meisten aromatischen Verbindungen, namentlich solche, die mehrere Ringsysteme enthalten, wie Naphthalin, Anthracen, Benzophenon, Dibenzyl, Chinolin zeigen nach kurzer Bestrahlung an Stelle des zuerst auftretenden Vorspektrums ein diskontinuierliches Phosphoreszenzspektrum, das Hauptspektrum, das meist aus einer größeren $\mathrm{Zahl}$ von schmalen hellen Streifen mit mehr oder minder ausgeprägten Hauptmaximis besteht. Ein wesentlich anderes, ebenfalls diskontinuierliches Spektrum, das Lösungsspektrum, zeigen dieselben Stoffe häufig, wenn ihre Lösungen in solchen Lösungsmitteln, die selbst nur kontinuierliche Phosphoreszenzspektra geben, untersucht werden. Das Vorspektrum ist entweder kontinuierlich oder besteht aus breiteren Banden.

Goldstein konnte nun zeigen, daß die Ent- 
stehung des diskontinuierlichen Hauptspektrums auf einer Umwandlung beruht, die die Substanzen unter der Einwirkung der Kathodenstrahlen erleiden, ganz ähnlich wie die Salze unter Bildung der Nachfarbe umgewandelt werden. Wird nämlich die Phosphoreszenz dieser Stoffe durch ultraviolettes Licht hervorgerufen, so entsteht im allgemeinen nur das Vorspektrum, sind sie aber zuvnr Kathodenstrahlen ausgesetzt worden, so zeigen sie, bisweilen noch nach monatelangem Aufbewahren bei Zimmertemperatur im ultravioletten Licht eine Phosphoreszenz, der das diskontinuierliche Hauptspektrum entspricht. Durch längere Bestrahlung der Stoffe mit sehr kurzwelligem ultravioletten Licht kann übrigens die gleiche Umwandlung wie durch die Kathodenstrahlen hervorgerufen werden.

Wie die Nachfarben durch Auflösen der Salze alsbald verschwinden, so verlieren die genannten aromatischen Verbindungen beim Schmelzen oder Lösen die Eigenschaft Hauptspektra zu geben momentan. $E_{s}$ ist also wahrscheinlich, daß auch die Veränderung dieser. Stoffe unter der Einwirkung der Kathodenstrahlen nicht chemischer, sondern physikalischer Natur sind. Sie stellen sich als reversible Lichtwirkungen dar, für die ich den Namen "Phototropie" in Vorschlag ge- bracht habe, als ich kurz nach den grundlegenden Goldsteinschen Entdeckungen verwandte Erscheinungen 'auffand, die bei gewissen organischen Verbindungen durch Bestrablung mit sichtbarem Licht hervorgerufen werden.

Im AnschluB an diesen Bericht sei an eine chemische Wirkung ultravioletten Lichtes erinnert, die zuerst von Lenard beobachtet, von Goldstein aber benutzt wurde, um die Chemie um ein sehr elegantes Verfahrem zur Gewinnung reinen Ozons zu bereichern. Dieses bildet sich bekanntlich, wie Lenard fand, wenn ultraviolettes Licht auf Sauerstoff ${ }^{*}$ einwirkt. Goldstein läßt nun in einem Geißlerschen Rohr, das bis $z u$ einem Quecksilberdruck von' mehreren Zentimetern mit Sauerstoff gefüllt ist und zu einem Teil in flüssige Luft eintaucht; die leuchtende Entladung vor sich gehen. Alsbald tritt Ozonbildung ein. Dieses kondensiert sich an den gekühlten GefäBwänden zu der bekannten tiefblauen Flüssigkeit, während der Gasdruck schnell abnimmt und in einer halben Minute bis auf $1 / 10$ Millimeter sinkt. LäBt man entsprechend Sauerstoff in das Rohr nachtreten, so kann man beliebige Mengen flüssiges Ozon gewinnen und den zugeführten Sauerstoff quantitativ in Ozon umwandeln.

\section{Das periodische System der chemischen Elemente im Lichte der jüngsten Kanalstrahlenforschung.}

Von Richard Swinne, Berlin-Friedenau.

\section{\$1. Einleitung.}

Die Zusammenfassung der chemischen Elemente zu natürlichen Gruppen auf Grund ähnlichen chemischen Verhaltens ergab gewisse regelmäßige Abstufungen bei Anordinung der Elemente nach steigenden (relativen) Atomgewichten und führte so vor etwa einem halben Jahrhundert zu den periodischen Systemen der Elemento von Lothar Meyer und Mendelejeff. Diese Anordnungen zeigten auch regelmäBige Abstufungen der physikalischen Eigenschaften der Elemente wie auch ihrer analogen Verbindungen. Immerhin ergaben die genaueren Atomgewichtsbestimmungen, da $B$ in einzelnen Fällen die durch die chemischen und sonstigen Eigenschaften bedingte Aufeinanderfolge der Elemente mit der Anordnung nach steigenden Atomgewichten in Widerspruch steht (A, K; Co, Ni; Te, J). Dies hätte schon damals Bedenken aufkommen lassen können; ob die Atomgewichte das maßgebende Charakteristikum für die Elementenfolge sind Die Entdeckung der Edelgase brachte keine Erschütterung des natürlichen Systems; diese fügten sich vielmehr sehr schön zwischen den elektropositiven Alkalimetallen und elektronegativen Halogenen ein. Die genauere Erforschung der Radioaktivität: lehrte zwar die Umwandelbarkeit gewisser Eisemente, verknüpfte aber durch die Verschiebungssätze die benachbarten Elemente mitein'ander und ließ einen Komplex gewisser chemischer wie auch physikalischer Eigenschaften im Falle eines Zerfalles spontan in einen anderen bestimmten Komplex von Eigenschaften übergehen. Die neu entdeckten Elemententypen konnten freie Plätze des periodischen Systems ausfüllen, wenngleich gleichzeitig der Begriff der Isotopie geprägt werden muBte, wodurch das Gewicht eines Atoms endgültig als nicht mehr maBgebend erkannt wurde, indem ,isobare" Elemente mit gleichem Gewicht ausgestattet sein, aber verschiedene, wenn auch benachbarte Plätze des natürlichen Systems einnehmen können.

Die im Anschluß an - die Erforschung der Streuung von $\alpha$-Teilchen entstandene Rutherfordsche Atomkerntheorie verlegte die Erscheinungen der Masse und der Radioaktivität in den positiv geladenen Atomkern, während die ihn umgebenden und neutralisierenden Elektronen die optischen Eigenschaften und insbesondere die äuBersten Elektronen die chemischen Eigenschaften bedingen sollten. Dio Kernladung erscheint hiernach als Charakteristikum eines bestimmten Elemententyps; sie ist der Ordnungszahl des Elements im natürlichen System igleichzusetzen und 